\title{
Tracing the coupled atomic shear and shuffle for a cubic to a hexagonal crystal transition
}

H.L. Wang, ${ }^{1}$ Y.L. Hao, ${ }^{1 *}$ S.Y. He, ${ }^{1}$ K. Du, ${ }^{1}$ T. Li, ${ }^{2,3}$ E.G. Obbard, ${ }^{4}$ J. Hudspeth, ${ }^{5}$ J.G. Wang, ${ }^{6}$ Y.D. Wang, ${ }^{7}$ Y. Wang, ${ }^{8}$ F. Prima, ${ }^{9}$ N. Lu, ${ }^{6}$ M.J. Kim, ${ }^{6}$ J.M. Cairney, ${ }^{2}$ S.J. Li, ${ }^{1}$ R. Yang $^{1}$

${ }^{1}$ Shenyang National Laboratory for Materials Science, Institute of Metal Research, Chinese Academy of Sciences, Shenyang 110016, China

${ }^{2}$ Australian Centre for Microscopy and Microanalysis, University of Sydney, Sydney, NSW 2006, Australia

${ }^{3}$ Institute for Materials and Materials Research Department \& ZGH, Ruhr-Universität Bochum, Universitätsstraße 150, 44780 Bochum, Germany

${ }^{4}$ Department of Electrical Engineering and Telecommunications, University of New South Wales, Sydney, NSW 2052, Australia

${ }^{5}$ European Synchrotron Radiation Facility, Grenoble 38043, France

${ }^{6}$ Department of Materials Science and Engineering, University of Texas at Dallas, Richardson, TX 75080, USA

${ }^{7}$ State Key Laboratory for Advanced Metals and Materials, University of Science and Technology, Beijing 100083, P.R. China

${ }^{8}$ Department of Materials Science and Engineering, Ohio State University, Columbus, OH 43210, USA

${ }^{9}$ PSL Research University, Chimie ParisTech-CNS, Institu de Recherche de Chimie Paris, Paris 75005, France

Tracing the rearranged atoms in the first-order phase transformation is unrealistic due to the discrete structure change. Here we report that, by tuning a nano-scale decomposition in a titanium alloy, the bcc crystal distorts successively toward the hcp crystal by keeping an orthorhombic symmetry. Thus, the shear-shuffle relationship is traced experimentally to enrich the well-known Burgers mechanism. Our results reveal also that the successive tuning on crystal structure at the atomic level leads to some novel properties which are unexpected from the discrete phase transformations.

*ylhao@imr.ac.cn. 
Solid-solid phase transformations typically undergo a discrete structure change from a parent to a product crystal via atomic shear and/or shuffle. The discrete nature raises a question of how to characterize these rearranged atoms. Thanks to the well-known orientation relationship of the parent and product crystals, the phase transformation pathways have been established geometrically, for example, the Burgers relationship/pathway of body centered cubic (bcc) and hexagonal close packed (hcp) crystals [1]. However, these geometrical models exclude the detailed information such as the sequence and correlation of the coupled atomic rearrangement [2-8]. Here gives a strategy to trace the structural change by tuning a discrete transformation into a continuous transition wherein the parent crystal is distorted successively toward the product crystal. This letter demonstrates for the first time that, by tuning a nano-scale concentration modulation via spinodal decomposition in a titanium alloy, the discrete bcc-hcp transformation can be continuous and the successive atomic shear and shuffle components are traceable by in-situ measurements.

The discrete bcc-hep transformation occurs in many solids such as $\mathrm{Fe}, \mathrm{Ti}, \mathrm{Zr}$ and their alloys $[9,10]$. The Burgers mechanism outlines a coupled shear and shuffle components [1-3]: (1) a shear component of a bcc $\{110\}$ plane, consisting of an elongation along [110] and compression along [100] perpendicular directions, to make perfect hexagons (Fig. 1a); and (2) a shuffle component of the adjacent $\{110\}$ planes to get a stacking sequence of the hcp crystal (Fig. 1b). The former results in an increase of the lattice parameter b/a ratio from 1.414 of the bcc crystal to 1.732 of the hcp crystal, while the ideal hcp crystal forms as the $c / a$ ratio increases from 1.414 to 1.633 . The latter leads to the Wyckoff position $y$ coordinate decreasing from 0.25 to 0.167 . Our measurements will provide solid evidence that these values vary continuously from the bcc to hcp, in sharp contrast with the sudden changes observed in the discrete transformations [4-6].

The above exploration was inspired by the recently-developed multifunctional titanium alloys with high strength and low elastic modulus $[11,12]$. Their continuous nonlinear deformation has attracted great attention and some reversible mechanisms besides the thermoelastic martensitic transformation (MT) have been proposed [13-16]. However, these have been challenged because a typical reversible MT, the $\beta$ (bcc) to $\alpha^{\prime \prime}$ (orthorhombic) MT, has been detected [15-17]. Our recent 3D atom probe tomography (APT) analysis of Ti2448 alloy showed that it passes through an isostructural spinodal decomposition [18], which creates a nano-scale architecture consisting of the interpenetrated $\mathrm{Nb}$-rich and Nb-lean domains. Since the Nb-rich domains are more stable, producing an elastic confinement 
on the MT in the neighboring Nb-lean domains, a nano-scale elastically confined MT mechanism was proposed [18]. Aided by the elastic confinement, the alloy is multifunctional over much wider temperature range than the typical shape memory alloys such as NiTi, for example, it exhibits the superelasticity from $4.2 \mathrm{~K}$ to $500 \mathrm{~K}$ and fully tunable thermal expansion, from positive, through zero, to negative, from $4.2 \mathrm{~K}$ to $625 \mathrm{~K}[18]$.

In this letter, we show that the spinodal decomposition is enhanced gradually as the alloy is heated and aged in the $\beta$ (bcc) and $\alpha$ (hcp) two-phase field. Both in-situ and ex-situ measurements reveal that, under the elastic confinement of the $\mathrm{Nb}$-rich $\beta$ domains, the $\mathrm{Nb}$-lean $\beta$ domains pass through a successive crystal distortion from bcc to hcp via the coupled atomic shear and shuffle by keeping an orthorhombic symmetry. Thus, the shear-shuffle relationship outlined by the Burgers mechanism is traced in experiment. Additionally, the above successive tuning on crystal structure at the atomic level leads to some novel properties which are unexpected from the discrete phase transformations.

The alloy has a nominal composition Ti-15Nb-2.5Zr-4Sn in atomic percent and is abbreviated as Ti2448 from its weight percent composition Ti-24Nb-4Zr-8Sn. An ingot was hot-forged at $850{ }^{\circ} \mathrm{C}$ and hot-rolled at $800{ }^{\circ} \mathrm{C}$ to $12 \mathrm{~mm}$ in diameter. The ingot has actual composition of $15.1 \mathrm{Nb}, 2.54 \mathrm{Zr}$, 3.99Sn and $0.48 \mathrm{O}$ in atomic percent and $24.2 \mathrm{Nb}, 3.96 \mathrm{Zr}, 8.10 \mathrm{Sn}$ and $0.13 \mathrm{O}$ in weight percent. In-situ synchrotron X-ray diffraction (SXRD) analysis was conducted on the high energy beamline ID15B of European Synchrotron Radiation Facility (ESRF) with a wavelength of $0.017048 \mathrm{~nm}$. A cylindrical sample of $3 \mathrm{~mm}$ in diameter and $10 \mathrm{~mm}$ in length was heated from 25 to $575{ }^{\circ} \mathrm{C}$ at a rate of $3{ }^{\circ} \mathrm{C} / \mathrm{min}$ and $2 \mathrm{D}$ diffraction patterns were recorded on a Pixium 4700 flat-panel detector. The patterns were analyzed with the whole pattern Rietveld method in the program package MAUD [19]. The APT samples were analyzed by a Cameca LEAP 4000X SI instrument and the data were reconstructed and analyzed using IVAS 3.6.6 ${ }^{\mathrm{TM}}$ software [20]. The TEM study was conducted using a probe-corrected JEOL ARM200F atomic resolution analytical electron microscope operated at $200 \mathrm{kV}$, equipped with a high-angle annular dark field (HAADF) detector and an energy dispersive $\mathrm{X}$-ray $(\mathrm{EDX})$ detector. Uniaxial tensile tests were performed at a strain rate of $2.5 \times 10^{-4} \mathrm{~s}^{-1}$ on an Instron 5582 universal test machine using rod sample with a gauge section $5 \mathrm{~mm}$ in diameter and 25 $\mathrm{mm}$ in length. The stress-strain curves were recorded by a strain extensometer.

It is clear from the integrated 1D SXRD profiles (Fig. 1c) that the heated alloy has a single bcc phase up to $375{ }^{\circ} \mathrm{C}$. As temperature increases further from 400 to $550{ }^{\circ} \mathrm{C}$, the orthorhombic (orth) 
phase, as indexed by these new and weak peaks, forms from the bcc matrix with larger volume at higher temperature. At $575{ }^{\circ} \mathrm{C}$, the orth phase is replaced by the hcp phase. Interestingly, the peak angles of the orth phase shift significantly until the formation of the hcp phase at $575{ }^{\circ} \mathrm{C}$. Meanwhile, the bcc phase peak angles do not change significantly. To clearly show the continuous peak shift of the orth phase, two sections in Fig. 1c are enlarged and presented in Fig. 1d.

From the SXRD profiles in Fig. 1c, the lattice parameters of three kinds of crystals were calculated and the variations of their ratios $(b / a$ and $c / a)$ with temperature are plotted in Fig. 1e. Similarly, the calculated Wyckoff position factors $(y)$ are presented in Fig. 1f. Since these new peaks in Fig. 1d become much weaker and broader at lower temperature, the early stage of bcc crystal distortion is untraceable. However, the slight distortion is estimated by extrapolating the measured data, which vary exponentially with temperature (see the curves in Figs. 1e and 1f). From this estimation we can conclude that the bcc crystal starts its distortion at $300{ }^{\circ} \mathrm{C}$, with a small fitting error of $\pm 2{ }^{\circ} \mathrm{C}$ for the $b / a$ and $c / a$ ratios and the factor $y$.

Aided by the experimental data in Figs. 1e and 1f, the shear-shuffle relationship can be established (Fig. 1g). Following the typical definitions [1-3], both shear and shuffle are set as zero for the initial bcc crystal and unity for the finial hcp crystal. The shear is defined by a unified change of $b / a$ ratio. To show the early stage of crystal distortion, the fitting data by the exponential curves in Figs. 1e and 1f are presented by a red curve in Fig. 1g. These results reveal that the shear and shuffle vary almost equally except weak shuffle and shear dominant at the start and the end respectively. This is in sharp contrast with the previous simulations to be strong shear or shuffle dominant [3,5,21].

The successively distorted bcc crystal can be detected by ex-situ TEM analysis. According to the Burgers mechanism, the shear component of a bcc $\{110\}$ plane also reduces the characteristic angle $\theta$ between the two (110) diagonals from $70.53^{\circ}$ to $60^{\circ}$ to make perfect hexagons [3]. As it can be seen in the selected area diffraction (SAD) patterns in Figs. 2a-2c of the samples aged for $2 \mathrm{~h}$ at several temperatures, the initial hot-rolled alloy has a single bcc phase with an angle $\theta$ of $70.5 \pm 0.3^{\circ}$ (Fig. 2a), the aging at $400{ }^{\circ} \mathrm{C}$ forms a small amount of the orth phase with an angle of $63.6 \pm 0.2^{\circ}$ (Fig. 2b) and the aging at $573{ }^{\circ} \mathrm{C}$ produces the hcp phase with an angle of $59.0 \pm 0.4^{\circ}$ (Fig. 2c). These results are summarized in Fig. 2d, clearly demonstrating that the characteristic angle $\theta$ changes continuously from the bcc to hcp crystals.

Previous investigations have shown that the hot-rolled Ti2448 alloy passes through a nano-scale 
isostructural spinodal decomposition [18]. Since the lattice mismatch induced by the $\mathrm{Nb}$ modulation is less than $0.05 \%$, which is at least one order of magnitude smaller than those reported in the typical decomposition alloys such as $\mathrm{FeCr}$ [22-26], these domains are equiaxed with size of 2 3 $\mathrm{nm}$ [18]. The study shows that the decomposition is incomplete and progresses gradually as the alloy is heated and aged in the bcc+hcp phase field. Coupled with the decomposition, the lattice mismatch goes up, for example, $\sim 8.3 \%$ as the alloy heated to $450{ }^{\circ} \mathrm{C}$ (calculated from the data in Fig. 1e). As a result, these domains grow into the lath morphology. Our microstructure analysis of the aged samples at the temperature range of 375 and $575{ }^{\circ} \mathrm{C}$ gives a consistent conclusion. Exampled by that aged at $450{ }^{\circ} \mathrm{C}$ for $2 \mathrm{~h}$, the HAADF image shows that the orth phase has a fine lath morphology (Fig. 3a) and the orth/bcc interface lacks sharp contrast (Fig. 3b), as confirmed by the inserted EDX spectrum that the repulsed $\mathrm{Nb}$ from the orth phase forms a pair of $\mathrm{Nb}$ concentration peaks near the orth/bcc interfaces. The 3D APT observations (Fig. 3c) reveal that the orth phase contains the finer intersectional laths with a thickness of less than $5 \mathrm{~nm}$. Consistent with the above EDX spectrum, both $\mathrm{Nb}$ and Ti change their concentrations continuously (Fig. 3d), a typical characteristic of spinodal decomposition [22,23]. Contributed by the nano-scale Nb modulation [27], the alloy is free of the $\omega$ phase, as evidenced by the in-situ SXRD profiles in Fig. 1c. This is in sharp contrast with the previous $\beta$ type titanium alloys. Since $\mathrm{Zr}$ and Sn, vary within the ranges of experimental error [18], the following focuses only on the nano-scale $\mathrm{Nb}$ modulation created by spinodal decomposition.

The isothermal orth phase in Fig. 3 can be athermal like the reversible MT. This novel behavior is confirmed by the cyclic tensile tests at room temperature. The stress-strain curves show that the aged samples with the isothermal orth phase exhibit a stress-induced reversibility (Fig. 4a) and their reversible strains decrease as the aging temperature increased (Fig. 4b). The novelty reveals that the successive crystal distortion, which is induced initially by an isothermal mechanism (Fig. 1f), can be continuous and reversible toward the direction to form the hcp crystal. This is consistent with the findings that the isothermal orth crystal with larger distortion, corresponding to smaller angle $\theta$ in Fig. 2d, exhibits weaker reversibility. So, the measured shear-shuffle relationship can apply to the reversible bcc-hcp and bcc-orth MTs. The former is common in pure Fe under the shock and high pressure [2-8] while the latter is the origin of the superelastic and shape memory effects of the recently developed bcc type titanium alloys [16].

Expected from the nano-scale elastic confinement and successive crystal distortion [18], the aged 
samples possess high ratio of strength $(\sigma)$ to modulus $(E)$ (Fig. 4c). The experimental data in Figs. $4 \mathrm{~b}$ and $4 \mathrm{c}$ confirm further that the orth crystal with smaller reversible strain exhibits lower $\sigma / E$ ratio due to stronger suppression on the successive crystal distortion. Additionally, the sample with a bcc+hcp microstructure as aged at $575{ }^{\circ} \mathrm{C}$ exhibits the lowest ratio of $\sim 1.5 \%$, which is still $\sim 50 \%$ higher than typical titanium alloys and 2 3 times higher than stainless steels. Such a strong contribution on strength is aided by the homogeneous nucleation of the hcp phase, a well-known merit of phase decomposition [26]. Since the decomposition occurs commonly in metallic materials, in particular of several ones observed recently in titanium alloys [28-31], they would have great potential to enhance both functional and mechanical properties like that in Ti2448 alloy.

In summary, we have shown through a model titanium alloy that the coupled atomic shear and shuffle components of the bcc-hcp phase transformation can be traceable experimentally as confined elastically by the nano-scale concentration modulation. The measured shear-shuffle relationship is helpful to understanding of the discrete phase transformations including the thermoealstic MT. The successive tuning on crystal structure provides new opportunity to explore novel properties, for example, the alloy exhibits unprecedented functional properties of the superelasticity and tunable thermal expansion across a wide temperature range and an excellent combination of mechanical properties such as flexible (low $E$ ), strong (high $\sigma / E$ ) and ductile at the same time.

The authors would like to thank MOST of China (2016YFC1102600), NSF of China (51571190, 51271180, 51631007, 51527801, 51390473, 51371177) for their support.

[1] W.G. Burgers, Physica 1 (1934) 561-586.

[2] M. Sanati, R.C. Albers, T. Lookman, A. Saxena, Phys. Rev. B 84 (2011) 1651-1659.

[3] J.B. Liu, D.D. Johnson, Physical Review B Condensed Matter 79 (2009) 897-899.

[4] K.J. Caspersen, A. Lew, M. Ortiz, E.A. Carter, Phys. Rev. Lett. 93 (2004) 115501.

[5] D.H. Kalantar, J.F. Belak, G.W. Collins, J.D. Colvin, H.M. Davies, J.H. Eggert, T.C. Germann, J. Hawreliak, B.L. Holian, K. Kadau, Phys. Rev. Lett. 95 (2005) 075502.

[6] A. Monza, A. Meffre, F. Baudelet, J.P. Rueff, M. D'Astuto, P. Munsch, S. Huotari, S. Lachaize, B. Chaudret, A. Shukla, Phys. Rev. Lett. 106 (2011) 247201.

[7] N.A. Zarkevich, D.D. Johnson, Phys. Rev. B 91 (2015) 174104. 
[8] M.P. Surh, L.X. Benedict, B. Sadigh, Phys. Rev. Lett. 117 (2016) 085701.

[9] E.W. Collings, The physical metallurgy of titanium alloys, American Society for Metals, Metals Park, Ohio, 1984.

[10]D. Bancroft, E.L. Peterson, S. Minshall, J. Appl. Phys. 27 (1956) 291-298.

[11]T. Saito, T. Furuta, J.H. Hwang, S. Kuramoto, K. Nishino, N. Suzuki, R. Chen, A. Yamada, K. Ito, Y. Seno, T. Nonaka, H. Ikehata, N. Nagasako, C. Iwamoto, Y. Ikuhara, T. Sakuma, Science 300 (2003) 464-467.

[12]Y.L. Hao, S.J. Li, S.Y. Sun, C.Y. Zheng, R. Yang, Acta Biomater. 3 (2007) 277-286.

[13]M.Y. Gutkin, T. Ishizaki, S. Kuramoto, I.A. Ovid'Ko, Acta Mater. 54 (2006) 2489-2499.

[14]Y. Hao, S. Li, B. Sun, M. Sui, R. Yang, Phys. Rev. Lett. 98 (2007) 216405.

[15]J. Cui, Y. Hao, S. Li, M. Sui, D. Li, R. Yang, Phys. Rev. Lett. 102 (2009) 045503.

[16]M. Tahara, H.Y. Kim, T. Inamura, H. Hosoda, S. Miyazaki, Acta Mater. 59 (2011) 6208-6218.

[17]E.G. Obbard, Y.L. Hao, R.J. Talling, S.J. Li, Y.W. Zhang, D. Dye, R. Yang, Acta Mater. 59 (2011) $112-125$.

[18] Y.L. Hao, H.L. Wang, T. Li, J.M. Cairney, A.V. Ceguerra, Y.D. Wang, Y. Wang, D. Wang, E.G. Obbard, S.J. Li, R. Yang, Journal of Materials Science \& Technology 32 (2016) 705-709.

[19]L. Lutterotti, S. Matthies, H.-R. Wenk, A.S. Schultz, J.W. Richardson, J. Appl. Phys. 81 (1997) 594-600.

[20]T. Li, D. Kent, G. Sha, L.T. Stephenson, A.V. Ceguerra, S.P. Ringer, M.S. Dargusch, J.M. Cairney, Acta Mater. 106 (2016) 353-366.

[21]C.X. Li, H.B. Luo, Q.M. Hu, R. Yang, F.X. Yin, O. Umezawa, L. Vitos, Solid State Commun. 159 (2013) 70-75.

[22]J.W. Cahn, J.E. Hilliard, The Journal of Chemical Physics 28 (1958) 258-267.

[23]J.W. Cahn, Acta Metall. 9 (1961) 795-801.

[24]A. Kelly, R.B. Nicholson, Precipitation Hardening, Progress in Materials Science, v.10, ed. by B. Chalmers, Macmillan, New York, 1963.

[25]A. G. Khachaturyan, Theory of structural transformations in solids, Dover Publications, New York, 2013.

[26]F. Findik, Mater Design 42 (2012) 131-146.

[27]H.L. Wang, S.A.A. Shah, Y.L. Hao, F. Prima, T. Li, J.M. Cairney, Y.D. Wang, Y. Wang, E.G. Obbard, S.J. Li, R. Yang, J. Alloys Compd., 700 (2017) 155-158. 
[28]S. Nag, A. Devaraj, R. Srinivasan, R.E.A. Williams, N. Gupta, G.B. Viswanathan, J.S. Tiley, S. Banerjee, S.G. Srinivasan, H.L. Fraser, R. Banerjee, Phys. Rev. Lett. 106 (2011) 245701.

[29]P. Barriobero-Vila, G. Requena, S. Schwarz, F. Warchomicka, T. Buslaps, Acta Mater. 95 (2015) 90-101.

[30] Y.M. Zhu, S.M. Zhu, M.S. Dargusch, J.F. Nie, Scripta Mater. 112 (2016) 46-49.

[31]Y. Zheng, D. Banerjee, H.L. Fraser, Scripta Mater. 116 (2016) 131-134. 

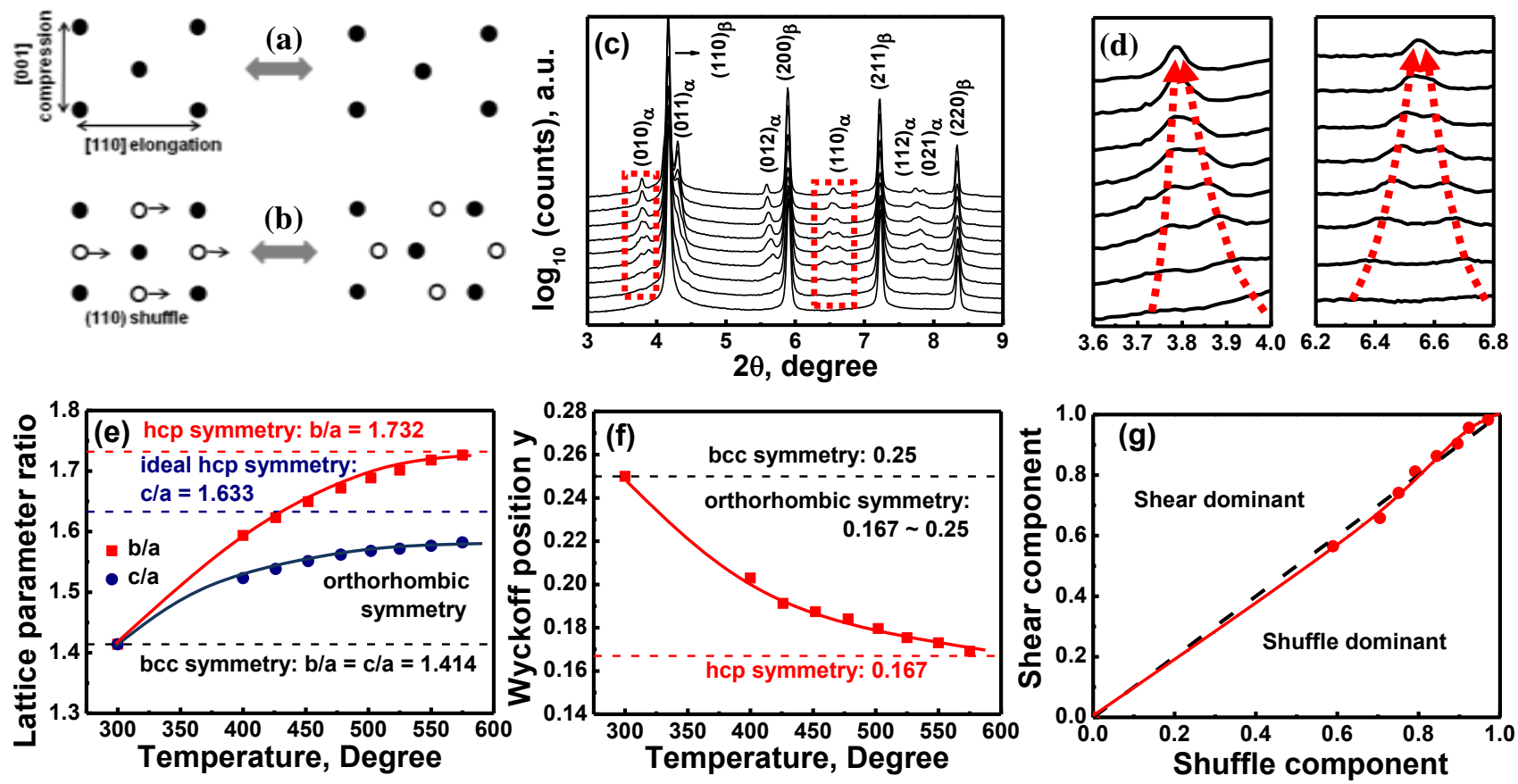

Fig. 1 In-situ SXRD analyses of the heated Ti2448 alloy. (a) and (b) Schematics of pure shear (a) and shuffle (b) components of the bcc-hcp transformation respectively. (c) Integrated 1D SXRD profiles recorded from 375 to $575{ }^{\circ} \mathrm{C}$ at an interval of $25^{\circ} \mathrm{C}$ (from bottom to top). (d) The enlarged sections indicated by the dotted rectangles in (c) show the new diffraction peaks and their significant shifts indicated by the curved arrows as temperature increased. (e) and (f) The continuously varied lattice parameter ratios (e) and the Wyckoff position factor (f) from the bcc crystal to the hcp crystal under the restriction of an orthorhombic symmetry. (g) The unified shear and shuffle relationship (the details see text). 

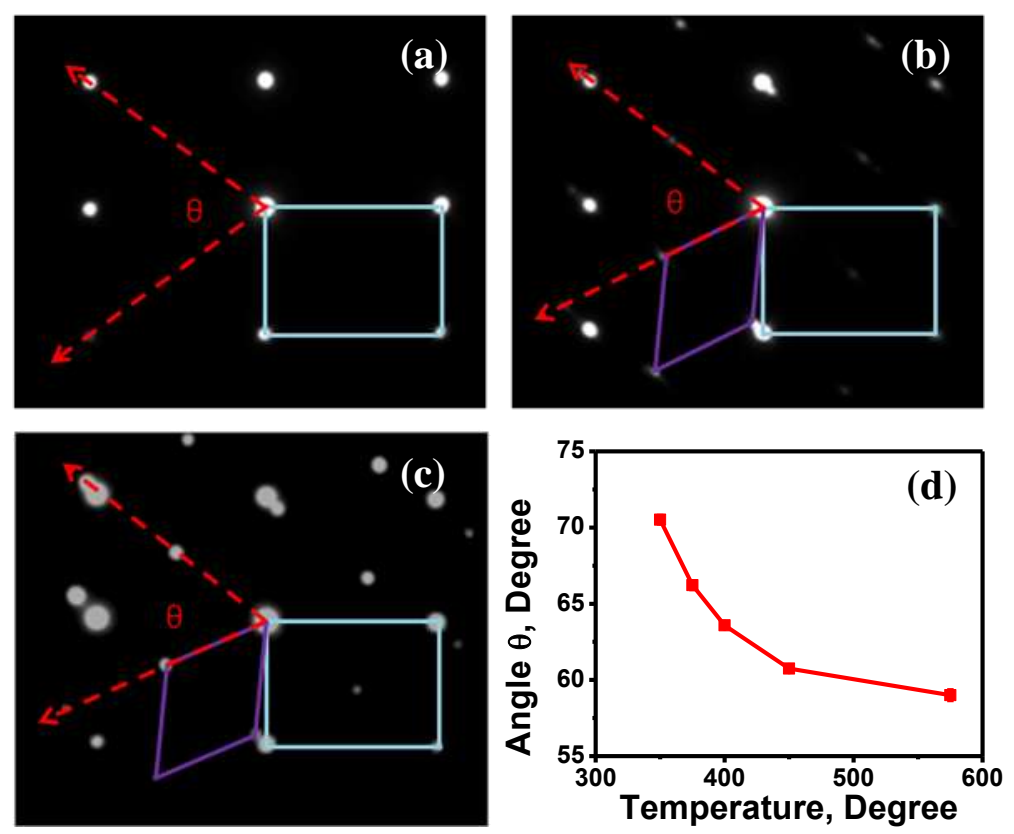

Fig. 2 Ex-situ TEM analyses of the aged Ti2448 alloy. (a-c) SAD patterns, taken along the [110] zone axis of the bcc $\beta$ phase, of the hot-rolled sample (a) and the aged samples at $400{ }^{\circ} \mathrm{C}$ (b) and $575{ }^{\circ} \mathrm{C}(\mathrm{c})$ for $2 \mathrm{~h}$, in which the bcc crystal is indicated by the light blue rectangle and the orth (b) and hcp (c) crystals are indicated by the purple rhombus. (d) Characteristic angle $\theta$ changes gradually from the bcc to hcp crystal_with the increase of aging temperature. 

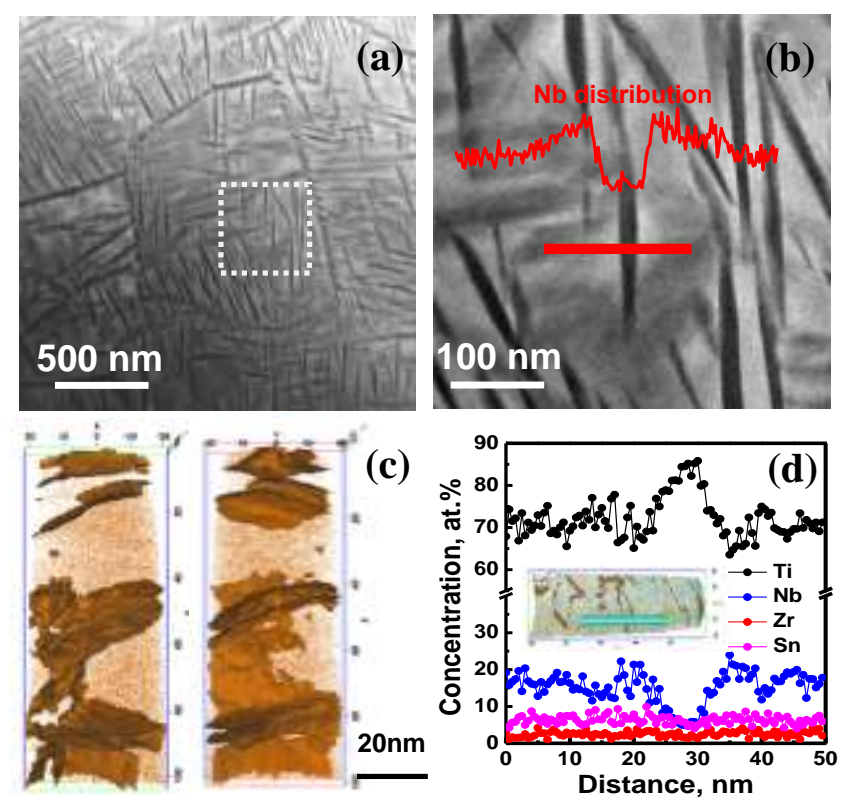

Fig. 3 The orth phase created by the spinodal decomposition in the aged Ti2448 alloy at $450{ }^{\circ} \mathrm{C}$ for 2h. (a) and (b) The HAADF images with low (a) and high (b) magnifications to show the lath morphology of the orth phase. The inset in (b) is the EDX scanning of $\mathrm{Nb}$ along the red line. (c) A pair of 3D APT iso-concentration surfaces of 9.5 at. $\% \mathrm{Nb}$, which are rotated $90^{\circ}$ to present the 3D lath morphology. (d) Concentration modulations along the line of the inserted 2D APT image. 


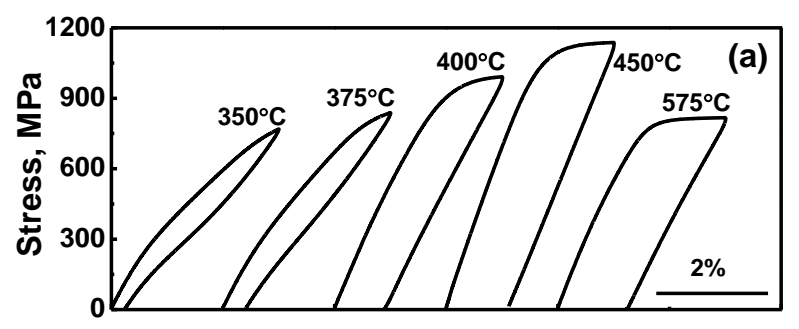

Strain, \%

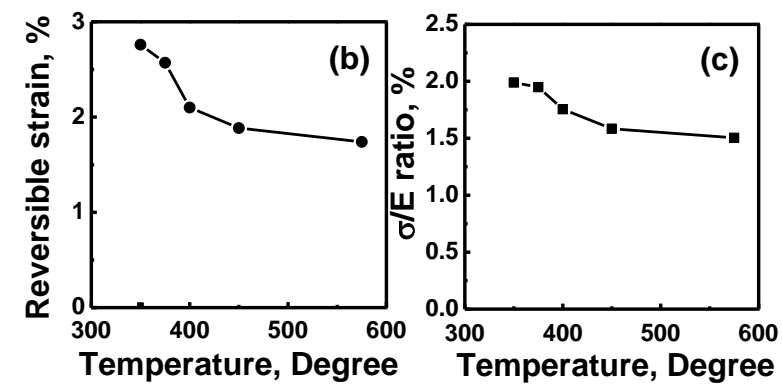

Fig. 4 Reversible deformation behavior of the samples aged for $2 \mathrm{~h}$. (a) Cyclic stress-strain curves with a total strain of 3\%. (b) and (c) Variations of the reversible strain $\left(\varepsilon_{\mathrm{r}}\right)$ and the ratio of strength to modulus $(\sigma / E)$ with the ageing temperature respectively. 

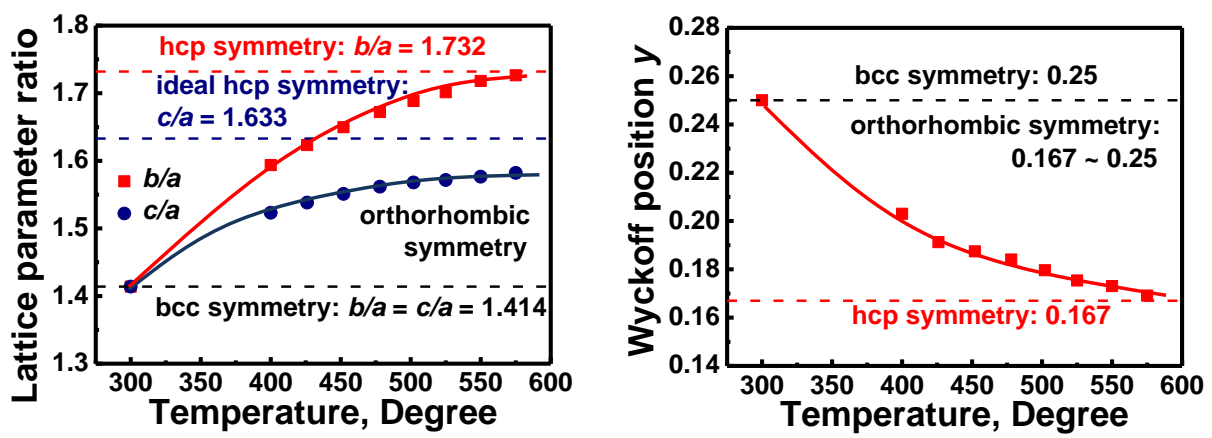